\title{
Doppler Velocimetry in the Human Fetus
}

\author{
Shoji Satoh \\ Maternity and Perinatal Care Unit, Oita Prefectural Hospital, Oita, Japan
}

Correspondence: Shoji Satoh, c/o Maternity and Perinatal Care Center, Oita Prefectural Hospital, Bunyo 476, Oita 870-8511 Japan, Phone: +81-97-546-7111, Fax: +81-97-546-0725, e-mail: satohs@ oitakenbyo.jp

\begin{abstract}
Doppler velocimetry has recently become an informative procedure for the real-time and noninvasive evaluation in fetal hemodynamics The analysis of flow velocity waveforms in various kinds of vessels has been made for not only clarifying fetal physiology and pathophysiology of various disorders but also for determining the most appropriate intervention from the viewpoint of the integrated management protocol. The unique change in both the arterial and venous flow in normal and compromised fetuses has been demonstrated. Clinical applications of the Doppler technique in monitoring the fetus are reviewed.

Keywords: Doppler velocimetry, Human fetus, Flow velocity waveforms, Arterial flow, Venous flow, Antenatal testing.
\end{abstract}

\section{INTRODUCTION}

With the advent of ultrasonographic technique, Doppler velocimetry has recently become an important and informative procedure for the real-time and noninvasive evaluation in fetal hemodynamics. The analysis of flow velocity waveforms has clarified fetal physiology and pathophysiology of various disorders. In these reports, functional evaluation using Doppler velocimetry is essential in fetal growth restriction (FGR) cases associated with uteroplacental insufficiency. I review clinical applications of the Doppler technique in monitoring the human fetus.

\section{DOPPLER VELOCIMETRY IN THE HUMAN FETUS}

Doppler velocimetry is a noninvasive technique for measuring the blood flow velocity by detecting ultrasound signals reflected by Doppler effect. In the human fetus, the application of this technique is two-fold; quantitative and qualitative analyses.

\section{Quantitative Evaluation of Fetal Blood Flow Volume by Doppler Velocimetry in the Human Fetus}

Historically, investigators utilized it for the calculation of circulating volume measurement in target organs by measuring vessel diameters or for the detection of absolute velocity in various fetal vessels. Velocity and volume measurement were performed in large vessels, such as descending or ascending aorta, pulmonary artery, umbilical vein or for the intracardiac flow. ${ }^{1-4}$ However, fairly large errors have been pointed out in the quantitative estimation of volume flow due to the possibility of inaccurate measurement of vessel diameters, the angle between ultrasound insonation and the vessel or the location of the sampling point. As the measurement of vessel diameters by the real-time ultrasonography particularly includes principal errors, the main utility of Doppler velocimetry has, to date, shifted to the analysis of blood flow shape.

\section{Qualitative Analysis of Doppler Velocity Waveforms}

Recent main trends have changed to qualitative analyses using the shape of flow velocity waveforms (FVWs). The detection of abnormal flow patterns and the determination of sampling points have recently become accessible with the help of color Doppler, a technique by which we can easily detect abnormal flow (e.g. regurgitant flow) or the existence of small vessels.

\section{Umbilical and Cerebral Arteries}

The most established target arteries in clinical applications in the human fetus are both the umbilical and the middle cerebral artery, because fetoplacental and cerebral circulation are essential and decisive for clinical management and fetal outcome. Although, a variety of parameters have been proposed for qualitative analyses in FVWs, the most common parameters are the resistance index (RI), pulsatility index (PI) and systolic/ diastolic ratio (S/D ratio), all of which are calculated by the peak-systolic flow, end-diastolic flow and mean flow velocities (Fig. 1). These parameters were originally used for detecting adult obstructive arterial diseases, in which they have elevated values as peripheral vascular resistance increases.

As both the fetoplacental and cerebral circulation develop, FVWs in the umbilical artery (UA) and middle cerebral arteries (MCA) change in unique manner. Many investigators have reported age-related changes of RI and PI in UA and MCA $\left(\mathrm{RI}_{\mathrm{UA}}, \mathrm{RI}_{\mathrm{MCA}}, \mathrm{PI}_{\mathrm{UA}}, \mathrm{PI}_{\mathrm{MCA}}\right)$, and RI and PI ratio between UA and MCA (RI $\left.\mathrm{RA}_{\mathrm{U} / \mathrm{MCA}}, \mathrm{PI}_{\mathrm{UA} / \mathrm{MCA}}\right)$. $\mathrm{RI}_{\mathrm{UA}}$ and $\mathrm{PI}_{\mathrm{UA}}$ demonstrated a gradual decrease as gestational age advanced. $\mathrm{RI}_{\mathrm{MCA}}$ and $\mathrm{PI}_{\mathrm{MCA}}$ showed a rather parabolic fashion in value with a peak around 30 to 31 weeks, indicating a gradual increase until 30 to 31 weeks of gestation and then a gradual decrease to term. $\mathrm{RI}_{\mathrm{UA} / \mathrm{MCA}}$ and $\mathrm{PI}_{\mathrm{UA} / \mathrm{MCA}}$ ratios decreased until 30 to 31 weeks of gestation and then increased to term. ${ }^{5} \mathrm{~A}$ uniform decrease in RI and PI in the umbilical artery through the whole gestational period suggest the gradual decrease in placental vascular 


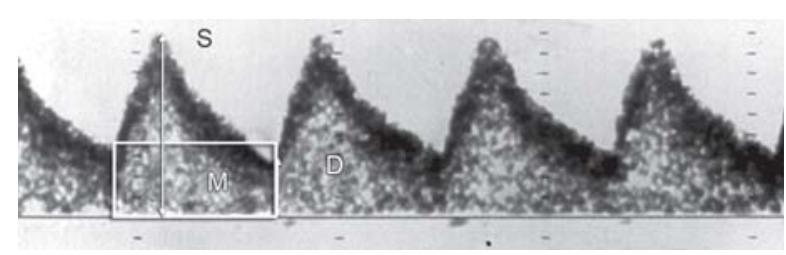

Fig. 1: Qualitative parameters for evaluating peripheral vascular resistance in the human fetus. $R I=(S-D) / S, P I=(S-D) / M$ $S / D$ ratio $=S / D . S=$ peak-systolic velocity, $D=$ end-diastolic velocity, $\mathrm{M}=$ mean velocity
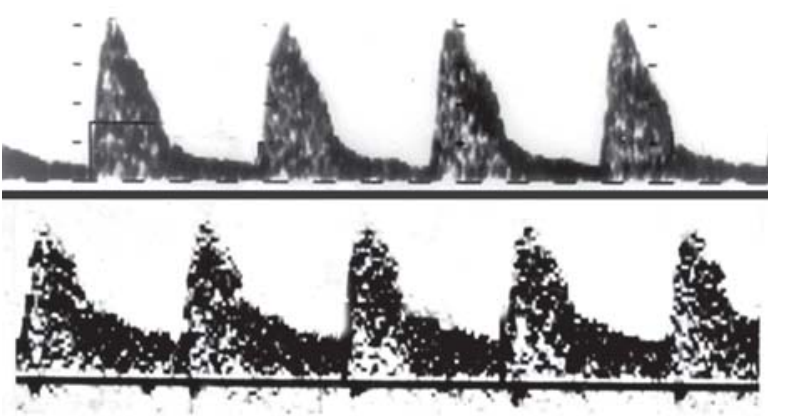

Fig. 2: Chronological change of FVW in the middle cerebral artery Above: Normal fetus (33 weeks of gestation). Bottom: FGR fetus (33 weeks of gestation)

resistance in normal development of fetoplacental circulation. ${ }^{6}$ On the other hand, the parabolic change of cerebral vascular resistance is speculated to be due to the rapid increasing volume in the cerebral vascular bed in both early and late gestational weeks, ${ }^{7}$ and the redistribution favoring blood supply to the brain, influenced by physiological $\mathrm{pO}_{2}$ reduction near term. ${ }^{8}$

Fetal growth restriction (FGR): Many investigations have pointed to the clinical usefulness of Doppler velocimetry in FGR. Its clinical application in this population is two-fold; whether it improves the detection rate of FGR, and whether it offers the possibility of monitoring the deterioration of the FGR fetus.

As for detection of impaired fetal growth, serial biometry by real-time ultrasonography is theoretically appropriate. FVW analysis in the umbilical artery or descending aorta, however, has fairly acceptable detectability for FGR by expressing hemodynamic aberration named 'brain sparing effect', which was first reported by Wladimiroff et al. ${ }^{9}$ They demonstrated low PI in the internal carotid artery, indicating the redistribution of blood supply to the brain as a high-priority organ in FGR cases (Fig. 2). We investigated the most effective parameter and cut-off values to detect FGR, using ROC curve. Our study included 170 FGR cases out of 684 high-risk singleton pregnancies, PI values in the umbilical artery measured within 2 weeks before delivery with the cut-off point of $1.5 \mathrm{SD}$, had the highest efficacy in detecting FGR with the sensitivity, specificity, positive predictive value, negative predictive value and accuracy of 60.6, 93.3, 75.2, 87.6 and 85.0\% respectively ${ }^{5}$ (Table 1). Several investigators have claimed increased efficacy by using the combined index of multiple arteries. Although the timing of the measurements, the kind of index, prevalence of FGR and risk levels differ in various studies; variation in the sensitivities, specificities and positive predictive values using FVW in the umbilical artery have been reported to be 41 to $100 \%, 65$ to $95 \%$ and 21 to $81 \%$ respectively, in high-risk pregnancies. $^{5,10-12}$

Hypoxia and/or acidosis: Fetal distress as manifested on fetal heart rate tracing, encompasses a wide range of etiology and pathophysiology, ranging from placental insufficiency, maternal disorders, fetal malformations to chromosomal aberrations. With the use of Doppler velocimetry, a relationship between cardiotocographic findings, which reflect fetal hypoxia or acidosis, and FVW changes has been established. We previously reported that FGR per se is associated with a chronic increase in the RI value in the umbilical artery, and that cerebral vascular resistance appears to decrease in conditions with abnormal fetal heart rate tracings. ${ }^{7}$ Scherjon et $\mathrm{al}^{13}$ studied the relationship between the umbilical artery PI/middle cerebral artery PI ratio and fetal outcome, and they concluded that the brain-sparing effect, indicated by elevated ratio, is a compensatory mechanism to prevent fetal brain hypoxia. Based on published reports, fetuses with hypoxia and/or acidosis are likely to show elevated placental vascular resistance and/or low cerebral vascular resistance, ${ }^{14}$ a phenomenon that is most apparent in FGR cases. Hecher et al have reported that the decrease in PI value in the middle cerebral artery directly correlates with the decrease in both venous blood $\mathrm{pO}_{2}$ and $\mathrm{pH} .{ }^{15}$ The main advantage of comparing the placental and the cerebral vascular resistance might be that the ratio includes both the possible existence of placental disease responsible for the change in maternal to fetal oxygen transfer and the cerebral hemodynamic consequences of blood gas change.

\begin{tabular}{|c|c|c|c|c|c|c|}
\hline & $R I_{\cup A}$ & $\mathrm{Pl}_{U A}$ & $R I_{M C A}$ & $P I_{M C A}$ & $R I_{U A / M C A}$ & $P_{U A / M C A}$ \\
\hline Cut-off (SD) & +1.25 & +1.5 & -1.0 & -1.0 & +1.0 & +1.0 \\
\hline Sensitivity (\%) & 62.4 & 60.6 & 41.8 & 40.6 & 64.7 & 60.0 \\
\hline Specificity (\%) & 91.5 & 93.3 & 89.1 & 87.3 & 85.7 & 88.3 \\
\hline PPV (\%) & 71.1 & 75.2 & 56.4 & 51.9 & 60.4 & 63.4 \\
\hline NPV (\%) & 87.8 & 87.6 & 82.0 & 81.4 & 87.8 & 86.8 \\
\hline Accuracy (\%) & 84.2 & 85.0 & 77.2 & 75.6 & 80.4 & 81.2 \\
\hline
\end{tabular}



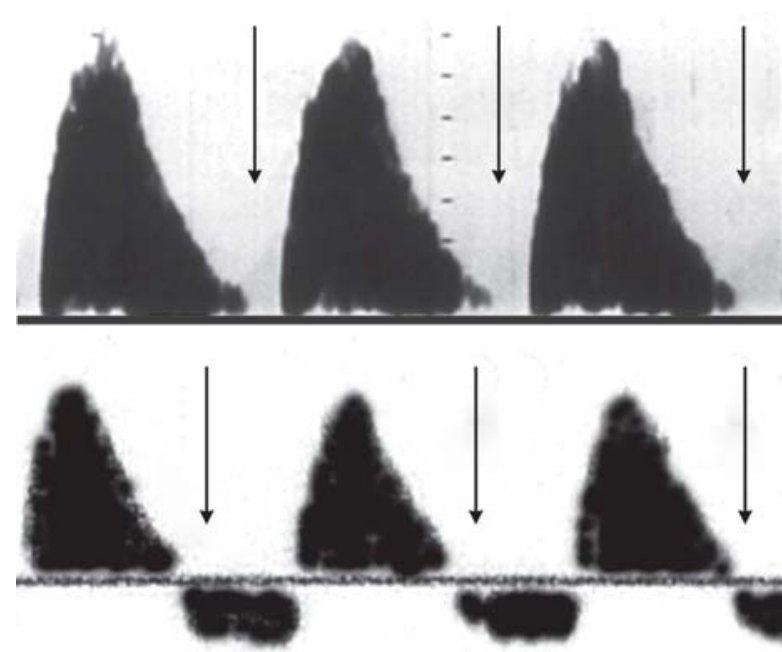

Fig. 3: Absent and reversed end-diastolic flow in the umbilical artery

Absent or reversed end-diastolic flow velocity in the umbilical artery: The absent or reverse diastolic flow (AREDF) in the umbilical FVW are likely to have strong relationship with poor fetal outcome (Fig. 3). It has been reported that AREDF is associated with high cesarean delivery rate, ${ }^{16-18}$ high incidence of pre-eclampsia, lower birth weight, lower gestational age at delivery, lower Apgar score and higher incidence of NICU admission. ${ }^{19,20}$ Because of its simplicity and acceptability of the correlation with outcome, this semiqualitative flow characteristic has been widely used as one of the clinical applications.

\section{Umbilical Vein}

The flow profile in the umbilical vein in normal fetuses is almost invariably continuous, although physiologically pulsatile flow pattern has been recognized in the first trimester. On the other hand, pathological pulsations have been reported as a probable result from the fetal heart failure in cases with hydrops fetalis, intrauterine growth restriction or hemolytic anemia. ${ }^{21,22}$ The main cause of the pulsatile flow is the reversed flow which originates from the pooling of blood, and the increase of obstructed venous inflow at the level of the vessels responsible for the venous return. ${ }^{23}$

\section{Inferior Vena Cava}

In inferior vena cava, the physiological and pathological flow pattern have been reported from the viewpoint of the reflection of right cardiac failure and the increase of the preload. The flow component in the inferior vena cava consists of two phases of forward flow during systolic and early diastolic period, followed by a reversed flow in late-diastolic phases (Fig. 4). It is known that the reversed flow component increases in some compromised conditions, such as persistent tachyarrhythmia, cardiogenic hydrops fetalis, the recipient in twin-twin transfusion and severely growth-restricted fetuses. ${ }^{24,25}$

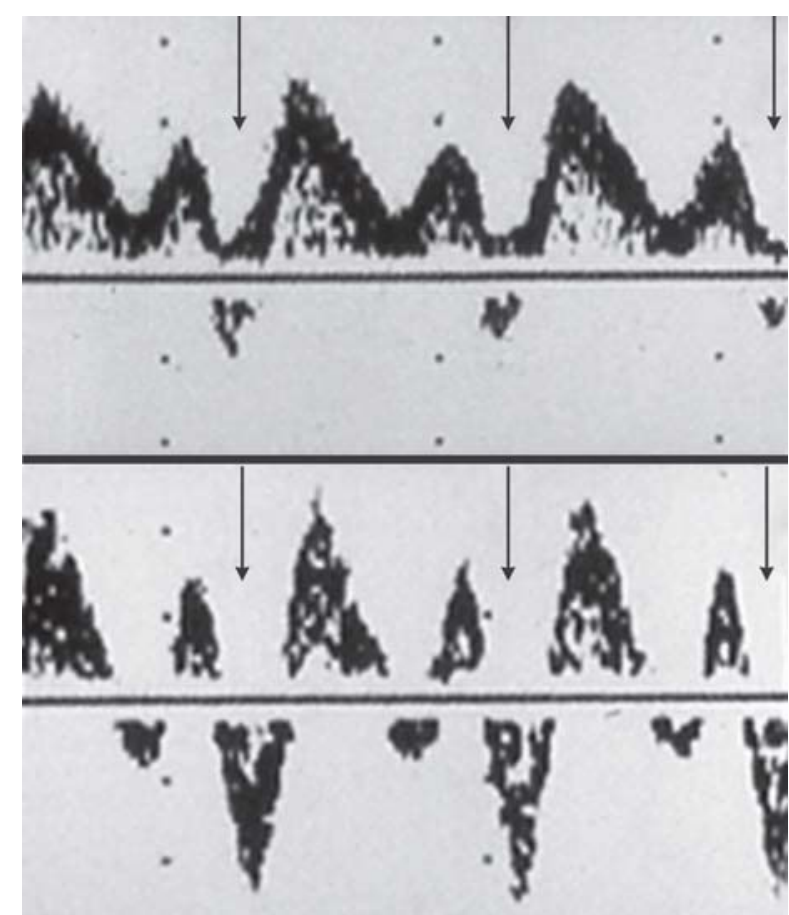

Fig. 4: FVW change in the inferior vena cava in FGR fetus. FVW has changed on admission at 32 weeks of gestation (left) to predominant reversed component at 34 weeks of gestation (right)

\section{Ductus Venosus}

The flow velocity waveform in the ductus venosus consists of two forward components. In contrast with inferior vena cava, no reverse flow can be detected in normal fetuses. It has been suggested that the decrease of the late-diastolic flow component precedes the fetal heart rate changes in placental insufficiency. ${ }^{26}$ In cases with rhesus isoimmunization, cardiogenic hydrops fetalis or severely compromised growth-restricted fetuses, reversed flow can be detected in late-diastole probably due to the elevation of the central venous pressure. ${ }^{27}$ Hence, the evaluation of the flow profile in the ductus venosus might be available for assessing whether the fetus is in 'decompensatory phase' or not.

\section{Integrated Testing by Doppler Velocimetry in FGR}

Many procedures have been utilized for the assessment of fetal well-being or compromise and, to date, the most popular and real-time technique in determining clinical management is fetal heart rate monitoring. Based on the fact that abnormal FVWs in the umbilical and/or intracranial artery correlate with poor fetal or neonatal outcome, the possibility of utilizing Doppler velocimetry as a parameter for indicating necessity or timing of delivery arises. Arduini et al reported high variation between 1 and 26 days for the time interval between the appearance of AREDF and abnormal fetal heart rate patterns. ${ }^{28}$ We also retrospectively reviewed the interval between the observation of the aberration of the flow waveform indices and subsequent 
fetal distress. Around 35 out of 52 cases with subsequent cardiotocographic findings of fetal distress had abnormal indices of $\mathrm{PI}_{\mathrm{UA} / \mathrm{MCA}}$ ratio, which lasted until delivery and revealed a wide range from 1 hour to 33 days between the first detection of abnormal flow pattern and fetal deterioration. ${ }^{5}$ Therefore, the results obtained by investigators indicate that the appearance of abnormal FVW is a warning sign necessitating intensive monitoring of fetal well-being. However it is difficult to accurately predict the most appropriate time of delivery. It must also be emphasized that termination of pregnancy should not be recommended based on abnormal FVWs alone, especially when fetuses are premature.

There are several prospective trials comparing Doppler FVW assessment with conventional testing, such as NST, biophysical profile or fetometry, to no use of Doppler information. ${ }^{29,30}$ Although their variables vary due to different patient population, they reach the same conclusion that Doppler examination does not greatly improve outcome as measured by gestational age at delivery, birth weight, cesarean delivery rate and NICU admissions. However, it might be reasonable to use Doppler FVW analysis as a screening for detecting high-risk fetuses in large pregnant populations, in combination with other ultrasonographic procedures, because there is adequate evidence of a significant relationship between abnormal FVWs and fetal deterioration.

To date, the evaluation of the fetal venous flow, in addition to the arterial flow profile, has been focused under the assumption that the hemodynamic aberration in venous circulation might demonstrate the decompensatory cardiac status. As for the venous circulation, the increase in reverse flow during atrial contraction in inferior vena cava, fluctuating flow in the umbilical vein and notching formation in FVWs in ductus venosus have been identified, ${ }^{31-33}$ which probably indicate increased atrial contractility and elevated venous pressure. All these phenomena are explained by possible increase of truncal and placental vascular resistance, resulting in the increase in afterload and blood flow redistribution to the brain. Mari and Picconi ${ }^{34}$ reported a longitudinal study in 10 FGR fetuses followed from the time the diagnosis of FGR was made up to delivery. Based on the chronological tendency of the pathophysiological blood flow deviation from 'arterial' to 'venous' branch, they have postulated that the last changes occurring in the cardiovascular system in severe FGR are right cardiac failure followed by left cardiac failure. Turan et $\mathrm{al}^{35}$ have integrated the chronological change of FVWs, the conventional biometry in FGR fetuses and proposed the management algorithm (Table 2). The algorithm is based on the ability to perform arterial and venous Doppler as well as a full five-component biophysical profile score. Their concept that the change of the amniotic fluid volume, the deterioration of the biophysical profile score or FVW in the umbilical artery is likely to precede FVW changes in the ductus venosus and/or the umbilical vein is very excellent.

\begin{tabular}{|c|c|c|}
\hline Antenatal testing & Interpretation & Action \\
\hline $\begin{array}{l}\text { Positive UA EDV } \\
\text { Normal MCA and veins } \\
\text { Max AF pocket }>2 \mathrm{~cm} \\
\text { BPS } \geq 8 / 10\end{array}$ & $\begin{array}{l}\text { Asphyxia extremely rare } \\
\text { Increased risk for intrapartum distress }\end{array}$ & $\begin{array}{l}\text { Deliver only for obsetric factors } \\
\text { BPS - weekly } \\
\text { Doppler - every other week }\end{array}$ \\
\hline $\begin{array}{l}\text { Blood flow redistribution } \\
\text { Positive UA EDV } \\
\text { Brain sparing, normal veins } \\
\text { Max AF pocket }>2 \mathrm{~cm} \\
\text { BPS } \geq 8 / 10, \text { AFV normal }\end{array}$ & $\begin{array}{l}\text { Hypoxemia possible, asphyxia rare } \\
\text { Increased risk for intrapartum distress }\end{array}$ & $\begin{array}{l}\text { > } 38 \text { weeks, consider delivery } \\
\text { BPS - twice weekly } \\
\text { Doppler - twice weekly }\end{array}$ \\
\hline $\begin{array}{l}\text { Significant blood flow redistribution } \\
\text { UA A/REDV, normal veins } \\
\text { BPS } \geq 8 / 10 \\
\text { Max AF pocket }<2 \mathrm{~cm}\end{array}$ & $\begin{array}{l}\text { Hypoxemia common } \\
\text { Acidemia or asphyxia possible } \\
\text { Onset of fetal compromise }\end{array}$ & $\begin{array}{l}>34 \text { weeks: deliver } \\
<32 \text { weeks: antenatal steroids } \\
\text { BPS and Dopper }-2-3 \text { times weekly }\end{array}$ \\
\hline $\begin{array}{l}\text { Fetal compromise } \\
\text { Significant redistribution } \\
\text { Increased DV pulsatility } \\
\text { BPS } \geq 6 / 10 \\
\text { Max AF pocket }<2 \mathrm{~cm}\end{array}$ & $\begin{array}{l}\text { Hypoxemia common } \\
\text { Acidemia or asphyxia likely }\end{array}$ & $\begin{array}{l}>32 \text { weeks: deliver } \\
<32 \text { weeks: admit, steroids } \\
\text { individualize testing daily }\end{array}$ \\
\hline $\begin{array}{l}\text { Fetal decompensation } \\
\text { Compromise by above criteria } \\
\text { Absent or reversed DV a-wave } \\
\text { Pulsatile UV, BPS }<6 / 10 \\
\text { Max AF pocket }<2 \mathrm{~cm}\end{array}$ & $\begin{array}{l}\text { Cardiovascular instability } \\
\text { Metabolic compromise } \\
\text { Stillbirth imminent } \\
\text { High perinatal mortality }\end{array}$ & Deliver at tertiary care center \\
\hline
\end{tabular}

Max: maximum, A/REDV: absent/reversed end-diastolic velocity, BPS: biophysical profile score, DV: ductus venosus, MCA: middle cerebral artery, UA: umbilical artery, AF: amniotic fluid, EDV: end-diastolic velocity 


\section{CONCLUSION}

Doppler velocimetry is an informative procedure to tell us of the physiological and pathophysiological hemodynamic change in the real-time manner. From the viewpoint of the clinical application, the technique is regarded as an important procedure for determining the most appropriate intervention using the integrated management protocol, particulary in FGR. Although many investigators have clarified the utility and limitation, the technique has been spread and fixed as the useful procedure for clarifying fetal compromise as well as well-being.

\section{REFERENCES}

1. Eik-Nes SH, Marsal K, Brubakk AO, et al. Ultrasonic measurement of human fetal blood flow. J Biomed Engng 1982;4:28-36.

2. Gill RW. Pulsed Doppler with B-mode imaging for quantitative blood flow measurement. Ultrasound Med Biol 1979;5:223-35.

3. Kurjak A, Rajhvajn B (Jr). Ultrasonic measurements of umbilical blood flow in normal and complicated pregnancies. J Perinat Med 1982;10:3-16.

4. Wladimiroff JW, McGhie JS. Ultrasonic assessment of cardiovascular geometry and function in the human fetus. Br J Obstet Gynaecol 1981;88:870-75.

5. Banu AA. Doppler velocimetry in the umbilical and middle cerebral arteries in fetuses with intrauterine growth retardation or fetal distress. Fukuoka Acta Med 1998;89:133-44.

6. Veille JC, Ben-Ami M, Sivakoff M. Ranged-gated pulsed Doppler of the umbilical artery in human fetuses during normal pregnancies. Am J Perinat 1991;8:269-72.

7. Satoh S, Koyanagi T, Fukuhara M, et al. Changes in vascular resistance in the umbilical and middle cerebral arteries in the human intrauterine growth-restricted fetus, measured with pulsed Doppler ultrasound. Early Hum Dev 1989;20:213-20.

8. Soothill PW, Nicolaides $\mathrm{KH}$, Rodeck $\mathrm{CH}$, et al. Effect of gestational age on fetal and intervillous blood gas and acid-base values in human pregnancy. Fetal Ther 1986;1:168-75

9. Wladimiroff JW, van den Wijngaard JA, Degani S, et al. Cerebral and umbilical arterial blood flow velocity waveform in normal and growth restricted pregnancies. Obstet Gynecol 1987;69: 705-09.

10. Arduini D, Rizzo G, Romanini C, et al. Fetal blood flow velocity waveforms as predictors of growth retardation. Obstet Gynecol 1987;70:7-10

11. Dempster J, Mires GJ, Patel N, et al. Umbilical artery velocity waveforms: Poor association with small-for-gestational-age babies. Br J Obstet Gynaecol 1989;96:692-96.

12. Gudmundsson S, Marsal K. Umbilical and uteroplacental blood flow velocity waveforms in pregnancies with fetal growth retardation. Eur J Obstet Gynaecol 1988;27:187-96.

13. Scherjon SA, Smoders-DeHaas H, Kok JH, et al. The "brainsparing” effect: Antenatal cerebral Doppler findings in relation to neurologic outcome in very preterm infants. Am J Obstet Gynecol 1993;169:169-75.

14. Mari G, Deter RL. Cerebral artery flow velocity waveforms in normal and small-for-gestational-age fetuses. Am J Obstet Gynecol 1992;166:1262-70.

15. Hecher K, Campbell S, Doyle P, et al. Assessment of fetal compromise by Doppler ultrasound of the fetal circulation. Circulation 1995;91:129-38.
16. Brar HS, Platt LD. Reverse end-diastolic flow velocity on umbilical artery velocimetry in high-risk pregnancies: An ominous finding with adverse pregnancy outcome. Am J Obstet Gynecol 1988;159:559-61.

17. McParland P, Steel S, Pearce JM. The clinical implications of absent or reversed end-diastolic frequencies in umbilical artery flow velocity waveforms. Eur J Obstet Gynecol Reprod Biol 1990;37:15-23.

18. Weiss E, Ulrich S, Berle P. Condition at birth of infants with previously absent or reverse umbilical artery end-diastolic flow velocities. Arch Gynecol Obstet 1992;252:37-43.

19. Battaglia C, Artini PG, Galli PA, et al. Absent or reversed enddiastolic flow in umbilical artery and severe intrauterine growth retardation. Acta Obstet Gynecol Scand 1993;72:167-71.

20. Divon NY, Girz BA, Lieblich R, et al. Clinical management of the fetus with markedly diminished umbilical artery end-diastolic flow. Am J Obstet Gynecol 1989;161:1523-27.

21. Gudmundsson S, Huhta JC, Wood DC, et al. Venous Doppler ultrasonography in the fetus with non-immune hydrops. Am J Obstet Gynecol 1991;164:33-37.

22. Indik JH, Chen V, Reed KL. Association of umbilical venous with inferior vena cava blood flow velocities. Obstet Gynecol 1991;77:551-57.

23. Huisman TWA. Doppler assessment of the fetal venous system. Semin Perinatol 2001;25:21-31.

24. Kanzaki T, Chiba Y. Evaluation of the preload condition of the fetus by inferior vena caval blood flow pattern. Fetal Diagn Ther 1990;5:168-74.

25. Rizzo G, Arduini D, Romanini C. Inferior vena cava flow velocity waveforms in appropriate- and small-for-getational-age fetuses. Am J Obstet Gynecol 1992;166:1271-80.

26. Hecher K, Hackeloer BJ. Cardiotocogram compared to Doppler investigation of the fetal circulation in the premature growthretarded fetus: Longitudinal observations. Ultrasound Obstet Gynecol 1997;9:152-61.

27. Huhta JC. Right ventricular function in the human fetus. J Perinat Med 2001;29:381-89.

28. Arduini D, Rizzo G, Romanini C. The development of abnormal heart rate patterns after absent end-diastolic velocity in umbilical artery: Analysis of risk factors. Am J Obstet Gynecol 1993;168:43-46.

29. Davies JA, Gallivan S, Spencer JAD. Randomized controlled trial of Doppler ultrasound screening of placental perfusion during pregnancy. Lancet 1992;340:1299-303.

30. Trudinger BJ, Cook CM, Giles WB, et al. Fetal umbilical artery velocity waveforms and subsequent neonatal outcome. $\mathrm{Br} \mathrm{J}$ Obstet Gynaecol 1991;98:378-84.

31. Indick JH, Chen V, Reed KL. Association of umbilical venous with inferior vena cava blood flow velocities. Obstet Gynecol 1991;77:551-57.

32. Rizzo G, Arduini D, Romanini C. Inferior vena cava flow velocity waveforms in appropriate and small for gestational age fetuses. Am J Obstet Gynecol 1992;166:1271-80.

33. Baschat AA, Harman CR. Antenatal assessment of the growth restricted fetus. Current Opinion in Obstet Gynecol 2001;13:1361-68.

34. Mari G, Picconi J. Doppler vascular changes in intrauterine growth restriction. Semin Perinat 2008;32:182-89.

35. Turan S, Miller J, Baschat AA. Integrated testing and management in fetal growth restriction. Semin Perinat 2008; 32 194-200. 